\title{
TROPICAL SEMIMODULES OF DIMENSION TWO
}

\author{
YA. SHITOV
}

\begin{abstract}
The tropical arithmetic operations on $\mathbb{R}$ are defined as $a \oplus b=\min \{a, b\}$ and $a \otimes b=a+b$. In the paper, the concept of a semimodule is discussed, which is rather ill-behaved in tropical mathematics. The semimodules $S \subset \mathbb{R}^{n}$ having topological dimension two are studied and it is shown that any such $S$ has a finite weak dimension not exceeding $n$. For a fixed $k$, a polynomial time algorithm is constructed that decides whether $S$ is contained in some tropical semimodule of weak dimension $k$ or not. This result provides a solution of a problem that has been open for eight years.
\end{abstract}

\section{§1. INTRODUCTION}

In tropical mathematics, the main object of study is the tropical semiring, that is, the set $\mathbb{R}$ of real numbers equipped with the tropical arithmetic given by the operations $a \oplus b=\min \{a, b\}$ and $a \otimes b=a+b$. The relationships between the usual and tropical mathematics can be described in terms of the Maslov dequantization [11. Tropical methods are useful in various applications related to algebraic geometry [13], combinatorial optimization [2], discrete event systems [9], etc. In this paper, we study the concept of a tropical semimodule, which is a tropical generalization of the classical notion of a linear space over a field.

There are a number of different important but nonequivalent ways to define the dimension of a tropical semimodule. The topological approach leads to the notion of the tropical rank, useful for various problems of geometry over fields with non-Archimedean valuation [6]. One can also define the dimension as the smallest cardinality of a generating set, and this approach leads to the study of tropical matrix factorizations (which are helpful for certain problems in combinatorial optimization [3]) and has interesting applications in computational biology [4]. In the paper, we investigate these two concepts of dimension and the relationships between them. To give a precise definitions of the notions under discussion, let us specify the notation to be used throughout the paper.

We denote the $(i, j)$ th entry of a matrix $A$ by $A_{i j}$, the submatrix of $A$ formed by the rows with labels $r_{1}, \ldots, r_{p}$ and columns with $c_{1}, \ldots, c_{q}$ by $A\left[r_{1}, \ldots, r_{p} \mid c_{1}, \ldots, c_{q}\right]$, the $i$ th row vector of $A$ by $A_{(i)}$, the $j$ th column vector of $A$ by $A^{(j)}$, and the transpose of $A$ by $A^{\top}$. We say that tropical matrices $B, C \in \mathbb{R}^{m \times n}$ coincide modulo scaling if there exist real numbers $\alpha_{1}, \ldots, \alpha_{m}, \beta_{1}, \ldots, \beta_{n}$ such that $B_{i j}=C_{i j}+\alpha_{i}+\beta_{j}$ for all $i$ and $j$.

The tropical operations with matrices and vectors are defined as the ordinary operations with + and $\cdot$ replaced by the tropical operations $\oplus$ and $\otimes$. In particular, a tropical linear combination of vectors $a_{1}, \ldots, a_{n} \in \mathbb{R}^{m}$ is a vector of the form $\left(\lambda_{1} \otimes a_{1}\right) \oplus \cdots \oplus$ $\left(\lambda_{n} \otimes a_{n}\right)$, where $\lambda_{1}, \ldots, \lambda_{n} \in \mathbb{R}$. The tropical linear span of vectors $a_{1}, \ldots, a_{n}$ is the set of all tropical linear combinations of these vectors.

A tropical semimodule $S \in \mathbb{R}^{n}$ (see [5]) is a subset closed under taking the tropical linear combinations of vectors in $S$. The topological dimension [6] of $S$ is the dimension

2010 Mathematics Subject Classification. Primary 15A03, 15A23, 15A80.

Key words and phrases. Tropical mathematics, linear algebra, computational complexity. 
of the set $S \in \mathbb{R}^{n}$ in the usual real topology. A finite subset $B$ of $S$ is called a weak basis 14 of $S$ if the tropical linear span of $B$ is $S$ and no vector in $B$ can be expressed as a tropical linear combination of the other vectors in $B$. If the tropical semimodule $S$ has a weak basis $B$, then every weak basis of $S$ coincides with $B$ up to scaling, and the cardinality of $B$ is then called the weak dimension of $S$ (see [14]).

The concept of the topological dimension is always well defined and makes sense for every tropical semimodule. Unfortunately, the same is not true for the weak dimension, and the following example provides us with a semimodule whose weak dimension is undefined.

Example 1.1 (see [1, Example 2.14]). The tropical semimodule generated by vectors $(-r, 0, r) \in \mathbb{R}^{3}$ (with $r$ running over $\mathbb{R}$ ) has no finite weak basis.

Example 1.1 shows that the concept of the weak dimension is rather ill-behaved, even if the topological dimension does not exceed three. Since the case of dimension one is trivial, we restrict our attention to semimodules of topological dimension not exceeding two. It turns out that with such a restriction, the semimodules demonstrate a much better behavior and their generating sets share a number of properties common with those of linear spaces over fields. In particular, we shall show that every tropical semimodule $S \subset \mathbb{R}^{n}$ has a weak basis of cardinality not exceeding $n$ if the topological dimension of $S$ does not exceed two. We also discuss algorithmic issues in the present paper, and we are interested in whether or not $S$ is contained in some tropical semimodule of fixed weak dimension $k$. We show that this problem can be solved in polynomial time, for any fixed $k$. We also discuss the linear algebraic consequences of our results.

\section{§2. Preliminaries}

Now, we provide a linear algebraic background of our considerations. The weak approach to defining concepts of dimension is related to the problem of factoring tropical matrices. This problem asks whether a given tropical matrix can be expressed as the product of an $m$-by- $k$ and a $k$-by- $n$ tropical matrix, for a given integer $k$. We introduce a precise definition of the concept of the factor rank, which emphasizes the essence of the problem in question and is also known as the combinatorial rank [3] and the Barvinok rank [6].

Definition 2.1 (see [1]). The factor rank of a tropical matrix $A \in \mathbb{R}^{m \times n}$ is the smallest integer $k$ for which there exist tropical matrices $B \in \mathbb{R}^{m \times k}$ and $C \in \mathbb{R}^{k \times n}$ satisfying $B \otimes C=A$.

The following well-known characterization of the factor rank follows directly from the definitions and will be useful for our considerations.

Lemma 2.2 (see [6]). The factor rank of a tropical matrix $A \in \mathbb{R}^{m \times n}$ is the smallest cardinality of a set $\alpha$ whose tropical linear span contains the columns of $A$.

In other words, the factor rank of a tropical matrix equals the smallest possible weak dimension of a semimodule containing the columns of $A$. Note that the word columns in Lemma 2.2 can be changed to rows because the factor rank is invariant under transposition. On the other hand, in general, the weak dimensions of the semimodules generated by the rows and columns of a given matrix are not the same, as the following example shows. 
Example 2.3 (see [1, Example 7.12]). The tropical semimodule spanned by the rows of the matrix

$$
A=\left(\begin{array}{lll}
1 & 0 & 1 \\
1 & 1 & 0 \\
0 & 0 & 1 \\
0 & 1 & 0
\end{array}\right)
$$

has weak dimension 4 , while the columns of $A$ span that of weak dimension 3.

The topological approach to defining the dimension leads one to the concept of the tropical rank, equal (see [6]) to the topological dimension of the tropical linear span of the rows of a matrix. The tropical rank admits a useful equivalent [6] combinatorial characterization [6], which can be described as follows. First, define the tropical permanent (also called the tropical determinant) of a tropical matrix $D \in \mathbb{R}^{n \times n}$ as

$$
\operatorname{perm}(D)=\min _{\sigma \in \mathcal{S}_{n}}\left\{D_{1, \sigma(1)}+\cdots+D_{n, \sigma(n)}\right\},
$$

where $\mathcal{S}_{n}$ stands for the symmetric group on $\{1, \ldots, n\}$. D is said to be tropically singular if the minimum in (2.1) is attained at least twice. The tropical rank of a tropical matrix $A$ is the largest integer $r$ such that $A$ has a non-singular $r$-by- $r$ submatrix.

It is easy to check that a tropical matrix has tropical rank one if and only if it has factor rank one (see [6]). It was also shown in [6] that the factor rank of a tropical matrix is always greater than or equal to its tropical rank. On the other hand, the following example shows that the factor rank can be arbitrarily large even for matrices with tropical rank two (in what follows, such matrices are simply called tropical rank-two matrices). We denote by $\lceil x\rceil$ the smallest integer greater than or equal to $x$ and by $\lfloor x\rfloor$ the largest integer not exceeding $x$.

Example 2.4 (see [6, Proposition 2.2]). Let $n \geq 2$ be an integer; consider the matrix

$$
C_{n}=\left(\begin{array}{ccccc}
a & 0 & 0 & \ldots & 0 \\
0 & a & 0 & \ldots & 0 \\
0 & 0 & a & \ldots & 0 \\
\vdots & \vdots & \vdots & \ddots & \vdots \\
0 & 0 & 0 & \ldots & a
\end{array}\right)
$$

with $a>0$. Then the tropical rank of $C_{n}$ equals 2 , and the factor rank equals the smallest integer $k$ for which $n \leq \frac{k !}{\left\lceil\frac{k}{2}\right\rceil !\left\lfloor\frac{k}{2}\right\rfloor !}$.

The computational problems related to tropical factorizations are also very interesting. In [3, Barvinok asked a question about the existence of a polynomial algorithm checking whether a given matrix has factor rank equal to a fixed integer $k$. In general, the answer is "no" even for $k=7$ (see [12]), and a version of this question restricted to matrices of small tropical rank was proposed in [6]. The case of tropical rank-one matrices is trivial [6], so in fact, the easiest interesting special case is that of matrices with tropical rank two. Even in this special case, the problem remained open. Develin, Santos, and Sturmfels ask Question (3b) in Section 8 of [6] as to whether a polynomial algorithm for factor rank exists or not if a given matrix is restricted to be of tropical rank not exceeding two.

Problem 2.5. Fix an integer $k$. Given a matrix $A$ with tropical rank not exceeding two, does there exist an algorithm deciding whether $A$ has factor rank equal to $k$ or not in polynomial time?

In this paper we give a positive answer to this question. On the other hand, the problem mentioned seems to be hard from a practical point of view. In particular, the 
algorithm presented in our paper for solving Problem 2.5] requires an amount of time triply-exponential in $k$ and is not indeed practical even for relatively small values of $k$.

The paper is organized as follows. In $\S 3$ we provide a useful characterization of ranktwo matrices up to scaling, and deduce a corollary that any tropical semimodule $S \subset \mathbb{R}^{n}$ has a finite basis if $S$ has topological dimension 2 . In $\S 4$, we provide a lower bound for the factor rank and discuss computational problems. In particular, we give a positive solution to Problem 2.5. We also show that a decision of whether or not a tropical twodimensional semimodule $S$ is contained in some tropical semimodule of weak dimension $k$ can be made in polynomial time, for every fixed $k$.

\section{§3. A GENERAL FORM OF RANK-TWO MATRICES AND BASES OF SEMIMODULES}

In this section we describe a general form of a tropical rank-two matrix, and then show that every tropical semimodule $S \subset \mathbb{R}^{n}$ with topological dimension not exceeding 2 has a generating set of cardinality at most $n$. Let us introduce the concept of a standard tropical rank-two matrix, which will be important for our characterizations.

Definition 3.1. A tropical matrix $A \in \mathbb{R}^{n \times n}$ is called a standard rank-two matrix if either $n=1$, or $A$ is a block matrix $\left(\begin{array}{cc}A^{\prime} & J_{1} \\ J_{2} & A^{\prime \prime}\end{array}\right)$ with $A^{\prime}$ and $A^{\prime \prime}$ standard rank-two matrices and $J_{1}$ and $J_{2}$ having all their entries equal to a real $\alpha$ which is the smallest entry in $A$.

Remark 3.2. It is not hard to check that standard rank-two matrices indeed have tropical ranks equal to 2 (with the only exception for a matrix whose all entries are equal, which is rank-one), but we do not present the proof because, in fact we do not need that result in our further considerations.

We proceed with a key lemma of the present section. Our technique generalizes that developed in Section 6 of [6] for dealing with tropical rank-two matrices. Recall that we denote by $A_{(i)}$ and $A^{(j)}$ the $i$ th row and $j$ th column of a matrix $A$, respectively.

Lemma 3.3. Let $A \in \mathbb{R}^{n \times m}$ be a matrix whose minimal entry appears in every row and every column, and let $J(t)$ denote the vector consisting of $t$ zeros. Assume that $A_{(i)} \oplus c \otimes J(m)=A_{\left(i^{\prime}\right)}$ implies $i=i^{\prime}$ for any $c \in \mathbb{R}$, and also that $A^{(j)} \oplus c \otimes J(n)=A^{\left(j^{\prime}\right)}$ implies $j=j^{\prime}$ for any $c$. If the tropical rank of $A$ does not exceed two, then there are permutations of rows and columns on $A$ such that the resulting matrix $\mathcal{A}$ is a standard rank-two matrix in which $\mathcal{A}_{i i}$ is a unique maximal element of both the ith row and ith column.

Proof. For $n=1$, the result is trivial, so we assume that $n>1$ and proceed by induction on $n$. Let $\alpha$ be the smallest number appearing in $A$. Up to permutations of rows and columns, we may assume that $J_{1}=A[1, \ldots, p \mid q+1, \ldots, m]$ is a maximal by inclusion submatrix consisting entirely of the $\alpha$ 's.

Step 1. If $q=0$, then we have $A_{(1)}=A_{(2)} \oplus \alpha \otimes J(m)$, which contradicts the assumption of the lemma. Therefore, we have $q>0$ and, similarly, $p<n$.

Step 2. Suppose that $A_{i^{\prime} j^{\prime}}>\alpha$, for some $i^{\prime}>p$ and $j^{\prime} \leq q$. Since $J_{1}$ is maximal by inclusion, there are $i^{\prime \prime} \leq p$ and $j^{\prime \prime}>q$ satisfying $A_{i^{\prime \prime} j^{\prime}}>\alpha$ and $A_{i^{\prime} j^{\prime \prime}}>\alpha$. By the assumption of the lemma, for some $i^{\prime \prime \prime}$ and $j^{\prime \prime \prime}$ we have $A_{i^{\prime \prime \prime} j^{\prime}}=A_{i^{\prime} j^{\prime \prime \prime}}=\alpha$, in which case the matrix $A\left[i^{\prime}, i^{\prime \prime}, i^{\prime \prime \prime} \mid j^{\prime}, j^{\prime \prime}, j^{\prime \prime \prime}\right]$ is tropically nonsingular, a contradiction.

So, we have $A=\left(\begin{array}{ll}A^{\prime} & J_{1} \\ J_{2} & A^{\prime \prime}\end{array}\right)$ with every entry of $J_{1}$ and $J_{2}$ equal to $\alpha$.

Step 3. Assume that $A_{i_{1} j_{1}}=\beta$ is a minimal number appearing in $A^{\prime}$ (that is, minimal over all $i_{1} \leq p$ and $\left.j_{1} \leq q\right)$. Suppose that there is $i_{2} \leq p$ such that $A_{i_{2} j_{2}}>\beta$ for every $j_{2} \leq q$. By the assumption of the lemma, there is $j_{3}$ for which $A_{i_{1} j_{3}} \neq A_{i_{2} j_{3}} \oplus \beta$, so that 
$j_{3} \leq q$ and $A_{i_{1} j_{3}}>\beta$. Now the matrix $A\left[i_{1}, i_{2}, n \mid j_{1}, j_{3}, m\right]$ is tropically nonsingular, a contradiction.

Therefore, every row (and, by a similar argument, every column) of $A^{\prime}$ has an entry equal to $\beta$.

Step 4. Assume that there are $c \in \mathbb{R}$ and different $i_{1}, i_{2} \in\{1, \ldots, p\}$ such that $A_{i_{1} j^{\prime}} \oplus c=$ $A_{i_{2} j^{\prime}}$ for every $j^{\prime} \leq q$. Then $c \geq \alpha$ because $A_{i_{2} j^{\prime}} \geq \alpha$, and so $A_{i_{1} j} \oplus c=A_{i_{2} j}$ for all $j \in\{1, \ldots, m\}$, because for $j^{\prime \prime}>q$ we have $A_{i_{1} j^{\prime \prime}}=A_{i_{2} j^{\prime \prime}}=\alpha$. This contradicts the assumption of the lemma.

So, we see that $A_{\left(i_{1}\right)}^{\prime} \oplus c \otimes J(q)=A_{\left(i_{2}\right)}^{\prime}$ is possible only if $i_{1}=i_{2}$. Similarly, we can show that $A^{\prime\left(j_{1}\right)} \oplus c \otimes J(p)=A^{\prime\left(j_{2}\right)}$ can be satisfied only if $j_{1}=j_{2}$.

Step 5 . Steps 3-4 show that the matrix $A^{\prime}$ (and thus also $A^{\prime \prime}$, by symmetry) satisfies the assumptions of the lemma. So we can use the inductive hypothesis for the matrices $A^{\prime}$ and $A^{\prime \prime}$. Therefore, we may assume that $A^{\prime}$ and $A^{\prime \prime}$ are standard rank-two matrices, and also that $A_{i^{\prime} i^{\prime}}$ (for $i^{\prime} \leq p$ ) is a unique maximum of the $i^{\prime}$ th row and $i^{\prime}$ th column of $A^{\prime}$, and that $A_{i^{\prime \prime} i^{\prime \prime}}\left(\right.$ for $i^{\prime \prime}>p$ ) is a unique maximum of the $i^{\prime \prime}$ th row and $i^{\prime \prime}$ th column of $A^{\prime \prime}$.

Step 6. Step 5 completes the proof of the lemma unless either $A^{\prime}$ or $A^{\prime \prime}$ is a 1-by-1 matrix with entry equal to $\alpha$. If this happens for $A^{\prime}$ (the case of $A^{\prime \prime}$ is the same up to row and column permutations), then we have $A_{(1)}=A_{(2)} \oplus \alpha \otimes J(m)$, which contradicts the assumption of the lemma.

Now we use Lemma 3.3 to obtain a characterization of general rank-two matrices.

Theorem 3.4. Let $A \in \mathbb{R}^{n \times m}$ be a matrix with tropical rank not exceeding 2. Assume that no row of $A$ can be expressed as a tropical linear combination of other rows, and no column of $A$ is a tropical linear combination of other columns. Then there is a standard rank-two matrix $\mathcal{A}$ equal to $A$ up to scaling and permutations of rows and columns and such that $\mathcal{A}_{i i}$ is a unique maximal element of both the ith row and ith column of $\mathcal{A}$.

Proof. Step 1. Over all $j \in\{1, \ldots, m\}$, we subtract $A_{1 j}$ from every entry of the $j$ th column of $A$, and so assume without loss of generality that $A_{1 j}=0$ for all $j$. Further, we can assume also that $A$ is nonnegative and that zero appears in every row, by subtracting the minimal entry of the $i$ th row of $A$ from every entry of that row.

Step 2. In particular, for every $i_{1}>1$ there is $j_{1}=j_{1}\left(i_{1}\right)$ satisfying $A_{i_{1} j_{1}}=0$. If also $A_{i_{2} j_{1}}>0$ for some $i_{2}$, Step 1 allows us to find $j_{2}$ for which $A_{i_{2} j_{2}}=0$.

Since the tropical sum of the rows from the second to last cannot be equal to the first row by the assumption of the theorem, we see that there is a $j_{3}$ for which $A_{i j_{3}}>0$ unless $i=1$. Now we see that the matrix $A\left[1, i_{1}, i_{2} \mid j_{1}, j_{2}, j_{3}\right]$ is tropically nonsingular, a contradiction.

Step 3 . Therefore, the column with index $j_{1}=j_{1}\left(i_{1}\right)$ consists entirely of zeros. Since the columns of $A$ are not allowed to coincide, we have $A_{i^{\prime} j^{\prime}}>0$ unless $i^{\prime}=1$ or $j^{\prime}=j_{1}$. In other words, up to permutations of rows and columns we have

$$
A=\left(\begin{array}{c|ccc}
a & 0 & \ldots & 0 \\
\hline 0 & & & \\
\vdots & & A^{\prime} & \\
0 & & &
\end{array}\right),
$$

where $a=0$ and the entries of $A^{\prime}$ are positive.

By adding a sufficiently small $\varepsilon>0$ to every entry of the first row and then subtracting $\varepsilon$ from every entry outside the first column, we can further assume that in fact $a>0$ in (3.1). 
Step 4. Consider some $c \in \mathbb{R}$ and a pair of different $i^{\prime}$ and $i^{\prime \prime}$. If $i^{\prime \prime}=1$, then by (3.1) we have $A_{i^{\prime} 1}<A_{i^{\prime \prime} 1}$, so that $A_{i^{\prime} 1} \oplus c \otimes J(m) \neq A_{i^{\prime \prime} 1}$. Therefore, $A_{\left(i^{\prime}\right)} \oplus c \otimes J(m)=A_{\left(i^{\prime \prime}\right)}$ can be true only when $i^{\prime \prime} \neq 1$, but then $A_{\left(i^{\prime}\right)} \oplus c \otimes A_{(1)}=A_{\left(i^{\prime \prime}\right)}$ which contradicts the assumption of the theorem.

Similarly, we can show that $A^{\left(j^{\prime}\right)} \oplus c \otimes J(n)=A^{\left(j^{\prime \prime}\right)}$ cannot occur for different $j^{\prime}$ and $j^{\prime \prime}$. Thus, the matrix $A$ satisfies the assumptions of Lemma 3.3 , and the result follows.

We point out an interesting corollary of Theorem 3.4

Corollary 3.5. Let $v_{1}, \ldots, v_{m}$ be vectors in $\mathbb{R}^{n}$ whose tropical linear span has topological dimension not exceeding two. If no vector among $v_{1}, \ldots, v_{m}$ can be expressed as a tropical linear combination of the other vectors, then $m \leq n$.

Proof. Let $V$ be an $n$-by- $m$ matrix whose $i$ th column is the vector $v_{i}$. Assume that the rows of $V$ with indices in a set $I \subset\{1, \ldots, n\}$ contain all the rows of $V$ in their tropical linear span; assume also that $I$ is also a minimal set having this property. Then no row of the matrix $V^{\prime}$ formed by the rows of $V$ indexed with the elements of $I$ is a tropical linear combination of the other its rows. Next, if there is $j$ such that $\bigoplus_{j^{\prime} \neq j}\left(\lambda_{j^{\prime}} \otimes V_{i j^{\prime}}\right)=V_{i j}$ for every $i \in I$, then these identities can be summed over $I$ with certain coefficients to obtain $\bigoplus_{j^{\prime} \neq j}\left(\lambda_{j^{\prime}} \otimes V_{i^{\prime} j^{\prime}}\right)=V_{i^{\prime} j}$ for any $i^{\prime} \notin I$ as well. This means that $v_{j}$ is a linear combination of other vectors among $v_{1}, \ldots, v_{m}$, and this is impossible by the assumption of the corollary. Therefore, no column of $V^{\prime}$ can be expressed as a tropical linear combination of the other its columns. Finally, the tropical rank of a matrix equals the topological dimension of the tropical linear span of its rows by [6, Theorem 4.2], whence $V^{\prime}$ has tropical rank not exceeding 2 . We see that $V^{\prime}$ satisfies the assumptions of Theorem 3.4, so that $m$ equals the cardinality of $I$.

Now we can show that every tropical semimodule $S \subset \mathbb{R}^{n}$ has a basis of cardinality not exceeding $n$ if the topological dimension of $S$ does not exceed 2 .

Theorem 3.6. Let $S \subset \mathbb{R}^{n}$ be a finitely generated tropical semimodule whose topological dimension does not exceed 2 . Then $S$ has a generating family of cardinality at most $n$.

Proof. Every such semimodule has a minimal generating set $G$, see [10, p. 153]. By Corollary 3.5, $G$ cannot have cardinality greater than $n$.

\section{$\S 4$. A LOWER BOUND FOR FACTOR RANK AND COMPUTATIONAL ISSUES}

In this section we prove that the factor rank of a tropical $n$-by- $n$ rank-two matrix $A$ is bounded from below by the minimal number $k$ satisfying $n \leq 2^{(k+2) !}$, provided no row of $A$ is a tropical linear combination of the other its rows and no column of $A$ is a tropical linear combination of the other its columns. We also present an algorithm checking whether or not the factor rank of a tropical rank-two matrix equals a fixed integer, and moreover, doing this in time polynomial in the size of the matrix. For the proof of our lower bound, the following special standard rank-two matrices will be helpful.

Definition 4.1. We say that a matrix $U \in \mathbb{R}^{n \times n}$ is a uniform tropical rank-two matrix if there is a real $\alpha$ such that $U_{i j}=\alpha$ if $i \neq j$ and $U_{i j}>\alpha$ otherwise.

Definition 4.2. Let $\alpha=\left(\alpha_{1}, \ldots, \alpha_{n}\right)$ and $\beta=\left(\beta_{1}, \ldots, \beta_{n}\right)$ be two real sequences satisfying $\alpha_{1}>\cdots>\alpha_{n}$ and $\alpha_{i}<\beta_{i}$ for every $i$. With these sequences, we associate the telescopic tropical rank-two matrix $S=S(\alpha, \beta) \in \mathbb{R}^{n \times n}$ defined by $S_{i j}=\min \left\{\alpha_{i}, \alpha_{j}\right\}$ if $i \neq j$ and $S_{i j}=\beta_{i}$ otherwise.

First, we prove lower bounds on the factor rank for uniform and telescopic matrices. 
Lemma 4.3. Assume that the factor rank of a uniform rank-two matrix of order $n$ does not exceed $k$. Then $n \leq \frac{k !}{\left\lceil\frac{k}{2}\right\rceil !\left\lfloor\frac{k}{2}\right\rfloor !}$.

Proof. For $n=1$ the result is trivial, so we assume that $n \geq 2$. Let $U$ be a matrix as in Definition 4.1, up to scaling we may assume that $\alpha=0$. Denote by $a$ the minimal diagonal entry of $U$ and consider the matrix $C$ occurring in Example 2.4. Let $D$ be a matrix such that $D_{i j}=a$ if $i \neq j$ and $D_{i j}=0$ otherwise. The relation $D \otimes U=C$ shows that the factor rank of $U$ is at least that of $C$, and the result follows.

Lemma 4.4. Assume that the factor rank of a telescopic matrix of order $n$ does not exceed $k$. Then $n \leq k$ !

Proof. The result is easy for $k=1$; we assume that $k>1$ and proceed by induction. Suppose by contradiction that our result is false, then there is a telescopic matrix $S=$ $S(\alpha, \beta)$ of order $n=k !+1$ and with factor rank not exceeding $k$. In other words, then we have $S=A \otimes B$ for some $A \in \mathbb{R}^{n \times k}$ and $B \in \mathbb{R}^{k \times n}$.

Step 1. Let $\lambda_{t}$ denote $\min \left\{A_{1 t}, \ldots, A_{n t}\right\}-\alpha(n)$. Since the tropical sum of the rows of $S$ is the vector $(\alpha(n), \ldots, \alpha(n))$, we have

$$
\min \left\{\lambda_{1}+B_{1 j}, \ldots, \lambda_{k}+B_{k j}\right\}=0 \text { for every } j \in\{1, \ldots, n\} .
$$

For every $t \in\{1, \ldots, k\}$, denote by $\Lambda_{t}$ the set of all $j \in\{1, \ldots, n\}$ for which $\lambda_{t}+B_{t j}$ is minimal over $\left\{\lambda_{1}+B_{1 j}, \ldots, \lambda_{k}+B_{k j}\right\}$; then $\Lambda_{1} \cup \cdots \cup \Lambda_{k}=\{1, \ldots, n\}$. By the pigeonhole principle, there is $\Lambda_{\tau}$ of cardinality $n^{\prime} \geq(k-1) !+1$. From (4.1) it also follows that $B_{\tau, j}=-\lambda_{\tau}$ for every $j \in \Lambda_{\tau}$.

Step 2. Assume that $\min _{t \neq \tau}\left\{A_{i t}+B_{t j}\right\} \neq S_{i j}$ for some different $i, j \in \Lambda_{\tau}$. Then, since $S=A \otimes B$, we have $A_{i \tau}+B_{\tau j}=S_{i j}=\min \{\alpha(i), \alpha(j)\} \leq \alpha(i)$. By the result of Step 1 , we obtain $A_{i \tau}+B_{\tau i} \leq \alpha(i)<\beta(i)=S_{i i}$, a contradiction with $S=A \otimes B$. So we see that $\min _{t \neq \tau}\left\{A_{i t}+B_{t j}\right\}=S_{i j}$ for all different $i$ and $j$ in $\Lambda_{\tau}$.

Step 3. Let $A^{\prime}$ denote the matrix formed by the rows of $A$ indexed with the elements of $\Lambda_{\tau}$ and the columns having indices different from $\tau$; let $B^{\prime}$ be the matrix formed by the columns of $B$ indexed with the elements of $\Lambda_{\tau}$ and the rows having indexes different from $\tau$; and let $S^{\prime}$ denote their tropical product $A^{\prime} \otimes B^{\prime}$. In these terms, the result of Step 2 means that $S_{i^{\prime} j^{\prime}}^{\prime}=\min \left\{\alpha\left(i^{\prime}\right), \alpha\left(j^{\prime}\right)\right\}$, for different $i^{\prime}$ and $j^{\prime}$. We also note that

$$
S_{i^{\prime} i^{\prime}}=\min _{t \neq \tau}\left\{A_{i t}+B_{t i}\right\} \geq \min _{t}\left\{A_{i t}+B_{t i}\right\}=S_{i i}>\alpha(i),
$$

so that, in fact, $S^{\prime}$ is a telescopic matrix. By the result of Step 1, the order of $S^{\prime}$ is at least $(k-1) !+1$, and since $S^{\prime}=A^{\prime} \otimes B^{\prime}$, the factor rank of $S^{\prime}$ does not exceed $k-1$. This contradicts the inductive assumption, completing the proof.

It will be useful to characterize the structure of standard rank-two matrices in terms of telescopic and uniform submatrices.

Lemma 4.5. Suppose that $A$ is a standard rank-two matrix having order $n$ and satisfying $A_{i i}>\max \left\{A_{i j}, A_{j i}\right\}$ for every different $i$ and $j$. Assume $A$ contains no uniform submatrix of order greater than $u$ and no telescopic submatrix of order greater than $t$. Then $n \leq u^{t}$. 
Proof. By the definitions, we have

$$
A=\left(\begin{array}{c|c|c|c|c}
A_{1} & J_{12} & J_{13} & \ldots & J_{1 s} \\
\hline J_{21} & A_{2} & J_{23} & \ldots & J_{2 s} \\
\hline J_{31} & J_{32} & A_{3} & \ldots & J_{3 s} \\
\hline \vdots & \vdots & \vdots & \ddots & \vdots \\
\hline J_{s 1} & J_{s 2} & J_{s 3} & \ldots & A_{s}
\end{array}\right),
$$

where every entry of the standard rank-two matrices $A_{1}, \ldots, A_{s}$ is greater than a real $\alpha$ and every entry outside $A_{1}, \ldots, A_{s}$ is equal to $\alpha$. Note that $s \leq u$ because otherwise $A$ has a uniform submatrix of order $u+1$. If $t=1$, then every $A_{i}$ has size $1 \times 1$, and the result follows. Otherwise, we assume that $t>1$ and proceed by induction on $t$.

Should $A\left[r_{1}, \ldots, r_{t} \mid c_{1}, \ldots, c_{t}\right]$ be a telescopic submatrix of $A_{i}$, we would take an $i^{\prime}$ that is not a row index for $A_{i}$, obtaining a telescopic submatrix $A\left[r_{1}, \ldots, r_{t}, i^{\prime} \mid c_{1}, \ldots, c_{t}, i^{\prime}\right]$ of $A$. Therefore, every $A_{i}$ has no telescopic submatrix of order greater than $t-1$, and by induction, $A_{i}$ has size not exceeding $u^{t-1}$.

Now we can prove a lower bound for the factor rank.

Theorem 4.6. Suppose a matrix $A \in \mathbb{R}^{n \times m}$ has factor rank not exceeding $k$ and tropical rank not exceeding two. If no row of $A$ can be expressed as a tropical linear combination of the other rows, and no column of $A$ is a tropical linear combination of the other columns, then $m=n \leq 2^{(k+1) !}$.

Proof. By Theorem 3.4, there is no loss of generality in assuming that $A$ is a standard rank-two matrix such that $A_{i i}$ is a unique maximal element of both the $i$ th row and $i$ th column of $A$. Next, should $A$ have a telescopic submatrix of order greater than $k$ !, then $A$ would have factor rank greater than $k$ by Lemma 4.4. Similarly, $A$ has no uniform submatrix of order greater than $2^{k}$ by Lemma 4.3. Now the result follows from Lemma 4.5.

The following corollary, relating the factor rank of submatrices to the factor rank of the entire matrix will be a key for our algorithmic results.

Corollary 4.7. Let $A$ be a matrix with tropical rank not exceeding two and factor rank greater than $k$. Then $A$ contains an $n$-by-m submatrix with factor rank exceeding $k$ such that $\max \{n, m\} \leq 2^{(k+2) !}$.

Proof. By Lemma 2.2, removing the row of a matrix may either decrease the factor rank by 1 or leave the factor rank unchanged. So, $A$ has a submatrix $A^{\prime}$ with factor rank equal to $k+1$. Note that removing a row that is a tropical linear combination of the other rows of a matrix will not change the factor rank by Lemma 2.2. Therefore, we can find a submatrix $A^{\prime \prime}$ (with the same factor rank as that of $A^{\prime}$ ) in which no row can be expressed as a tropical linear combination of the other rows, and no column of $A^{\prime \prime}$ is a tropical linear combination of the other columns. Now the result follows from Theorem 4.6 .

Now we are ready to provide a positive solution to Problem 2.5. We can construct an algorithm which is given a tropical rank-two matrix $A$ and checks whether or not $A$ has factor rank equal to an integer $k$ in time polynomial in the size of a matrix, for every fixed $k$.

Theorem 4.8. Fix an integer $k$. A decision whether or not a given matrix $A \in \mathbb{R}^{m \times n}$ with tropical rank not exceeding 2 has factor rank equal to $k$ can be made by a polynomialtime algorithm. 
Proof. By Corollary 4.7 it suffices to compute the factor ranks of submatrices of $A$ having at most $2^{(k+2) !}$ rows and at most $2^{(k+2) !}$ columns, so that the number of those submatrices does not exceed $(m n+m+n+1)^{2^{(k+2) !}}$. The factor rank of each of those submatrices can be computed in time bounded as a function of $k$ thanks to the quantifier elimination algorithm for the first-order theory of real addition with the order, see 8 .

In the following corollary, we assume that a tropical semimodule is defined by its generating family of cardinality not exceeding $n$. In fact, such a generating family exists for any semimodule of topological dimension two, see Theorem 3.6 .

Corollary 4.9. Fix an integer $k$. Given a family $v_{1}, \ldots, v_{m}$ of vectors generating a tropical semimodule $S$ of topological dimension not exceeding two, it can be decided in polynomial time whether or not $S$ is contained in a tropical semimodule of dimension $k$.

Proof. By Lemma 2.2, we need to check whether the matrix whose columns are $v_{1}, \ldots, v_{m}$ has factor rank not exceeding $k$. Thus, the result follows from Theorem 4.8.

As a final remark, we note that Theorem 4.8 admits no generalization to matrices of arbitrary tropical rank. In fact, it is an NP-hard problem [12] to decide whether or not the factor rank equals 7 even if a given matrix has tropical rank not exceeding 7 .

\section{REFERENCES}

[1] M. Akian, S. Gaubert, and A. Guterman, Linear independence over tropical semirings and beyond, Contemp. Math., vol. 495, Amer. Math. Soc., Providence, RI, 2009, pp. 1-38. MR2581511 (2011e:16085)

[2] F. Baccelli, G. Cohen, G. J. Olsder, and J. P. Quadrat, Synchronization and Linearity, An algebra for discrete event systems, John Wiley \& Sons, Ltd., Chichester, 1992. MR.1204266 (94b:93001)

[3] A. I. Barvinok, Two algorithmic results for the traveling salesman problem, Math. Oper. Res. 21 (1996), no. 1, 65-84. MR1385867 (97a:90102)

[4] M. Develin, The moduli space of $n$ tropically collinear points in $\mathbb{R}^{d}$, Collect. Math. 56 (2005), no. 1, 1-19. MR2131129 (2005m:55025)

[5] M. Develin and B. Sturmfels, Tropical convexity, Doc. Math. 9 (2004), 1-27. (electronic) MR2054977(2005i:52010)

[6] M. Develin, F. Santos, and B. Sturmfels, On the rank of a tropical matrix, Combinatorial and Computational Geometry, Math. Sci. Res. Inst. Publ., vol. 52, Cambridge Univ. Press, Cambridge, 2005, pp. 213-242. MR2178322 (2006g:15001)

[7] M. Einsiedler, M. Kapranov, and D. Lind, Non-Archimedean amoebas and tropical varieties, J. Reine Angew. Math. 601 (2006), 139-157. MR2289207 (2007k:14038)

[8] J. Ferrante and C. Rackoff, A decision procedure for the first order theory of real addition with order, SIAM J. Comput. 4 (1975), 69-76. MR0389572 (52:10403)

[9] S. Gaubert, Methods and applications of (max, +) linear algebra, STACS 97 (Lubek), Lecture Notes in Comput. Sci., vol. 1200, Springer, Berlin, 1997, pp. 261-282. MR.1473780 (98f:68205)

[10] J. S. Golan, Semirings and their applications, Kluwer Acad. Publ., Dordrecht, 1999. MR1746739 (2001c:16082)

[11] G. Litvinov and V. Maslov, The correspondence principle for idempotent calculus and some computer applications, Cambridge Univ. Press, Cambridge, 1998, pp. 420-443. MR.1608383(99c:16050)

[12] Ya. Shitov, The complexity of tropical matrix factorization, Adv. in Math. 254 (2014), 138-156. MR 3161095

[13] O. Viro, Dequantization of real algebraic geometry on logarithmic paper, European Congress of Math., Vol. 1, Progr. Math., vol. 201, Birkhäuser, Basel, 2001, pp. 135-146. MR1905317 (2003f:14067) 
[14] E. Wagneur, Moduloids and pseudomodules. I, Dimension theory, Discrete Math. 98 (1991), no. 1, 57-73. MR 1139597(92i:08003)

National Research University-Higher School of Economics, Myasnitskaya Ulitsa 20, MosCOW 101000, Russia

E-mail address: yaroslav-shitov@yandex.ru

Received 27/JUN/2013

Originally published in English 